\title{
Scalar and Vector Massive Fields in Lyra's Manifold
}

\author{
R. Casana, C. A. M. de Melo* and B. M. Pimentel \\ Instituto de Física Teórica, Universidade Estadual Paulista \\ Rua Pamplona 145, CEP 01405-900, São Paulo, SP, Brazil \\ E-mail: casanalift.unesp.br, cassiuseift.unesp.br \\ pimenteleift.unesp.br
}

\begin{abstract}
The problem of coupling between spin and torsion is analysed from a Lyra's manifold background for scalar and vector massive fields using the Duffin-Kemmer-Petiau (DKP) theory. We found the propagation of the torsion is dynamical, and the minimal coupling of DKP field corresponds to a non-minimal coupling in the standard Klein-Gordon-Fock and Proca approaches. The origin of this difference in the couplings is discussed in terms of equivalence by surface terms.
\end{abstract}

Fourth International Winter Conference on Mathematical Methods in Physics 09 - 13 August 2004

Centro Brasileiro de Pesquisas Físicas (CBPF/MCT), Rio de Janeiro, Brazil

${ }^{*}$ Speaker. 


\section{Introduction}

After Einstein's approach to gravitation, several others theories have been developed, as part of efforts to cure problems arising when the gravitational field is coupled to matter fields. In particular, the problem of spin coupling to gravitation has a central role in some recent years. The principal path to incorporate spin in geometrical theories of gravitation is the use of so called Riemann-Cartan geometry. This geometry has a nonsymmetric connection, in such a way that a new geometrical concept enters in scene: the torsion. However, analysing the Cauchy data, one can proof the torsion is a nonpropagating entity and therefore must be different of zero only in the interior of matter.

As soon as Einstein presented the General Relativity, Weyl [1] proposed a new geometry in which a new scalar field accompany the metric field and change the scale of length measurements. The aim was to unify gravitation and electromagnetism, but this theory was briefly refuted by Einstein because the nonmetricity had direct consequences over the spectral lines of elements which never has been observed.

After some more years, Lyra [2] has proposed a new geometry, with scalar field for scale changes, that respect the metricity condition. This theory was developed by Scheibe [2], Sen [3] and several others as an alternative to describe the gravitational field, and more recently has been applied to study viscous [4] and higher dimensional [5] cosmological models, domain walls [6], and several others applications. In context of spin-gravitational coupling, the importance of Lyra's geometry resides in the fact that the torsion is propagating.

On the other hand, to study the behaviour of scalar and vector massive fields in non-euclidean manifolds is extremely important in the context of astroparticle physics and unified theories since a great part of our knowledge about cosmological data and fundamental interactions is described by this type of field. A profitable manner of describing these fields is to use the Duffin-Kemmer-Petiau (DKP) theory. In DKP theory, both particles are described by only one field with a linear first order differential equation, very similar to Dirac equation. This similarity can be employed to facilitate the study of interactions between several fields, just as in General Relativity [7, 8] and EinsteinCartan spacetimes [9, 10]. However, in the last case is found that DKP theory is not equivalent to the correspondent Klein-Gordon-Fock (KGF) and Proca Lagrangians. Notwithstanding, the HarischChandra theory for massless DKP field [11] was extended to Riemann-Cartan manifold in such a way that a complete equivalence with KGF and Maxwell theories can be proved [12]. Therefore, the equivalence between DKP and the more usual theories is not trivial, and the question of what is the most fundamental theory arises. Evidently, only a very accurate experiment could decide.

Here, we propose look for coupling of spin 0 and 1 massive fields and torsion in Lyra manifold via DKP formalism. A good introduction to DKP theory can be found in [13, 14]. In section 2 we present the essential elements of Lyra geometry, and in the subsequent sections the coupling of DKP field with curvature and torsion in this manifold as well as a comparison between the results of the more usual KGF and Proca formalisms. Finally, in the last section we make some comments on the results. 


\section{The Lyra geometry}

The Lyra manifold [2] is defined given a tensor metric $g_{\mu v}$ and a positive definite scalar function $\phi$ which we call the scale function. In Lyra geometry, one can change scale and coordinate system in an independent way, to compose what is called a reference system transformation: let $M \subseteq \mathbb{R}^{N}$ and $U$ an open ball in $\mathbb{R}^{n},(N \geq n)$ and let $\chi: U \curvearrowright M$. The pair $(\chi, U)$ defines a coordinate system. Now, we define a reference system by $(\chi, U, \phi)$ where $\phi$ transforms like

$$
\bar{\phi}(\bar{x})=\bar{\phi}(x(\bar{x}) ; \phi(x(\bar{x}))), \quad \frac{\partial \bar{\phi}}{\partial \phi} \neq 0
$$

under a reference system transformation.

In the Lyra's manifold, vectors transform as

$$
\bar{A}^{v}=\frac{\bar{\phi}}{\phi} \frac{\partial \bar{x}^{v}}{\partial x^{\mu}} A^{\mu}
$$

In this geometry, the metric connection is

$$
\tilde{\Gamma}_{\mu v}^{\rho} \equiv \frac{1}{\phi} \stackrel{\circ}{\Gamma}_{\mu v}^{\rho}+\frac{1}{\phi}\left[\delta_{\mu}^{\rho} \partial_{v} \ln \left(\frac{\phi}{\bar{\phi}}\right)-g_{\mu v} g^{\rho \sigma} \partial_{\sigma} \ln \left(\frac{\phi}{\bar{\phi}}\right)\right], \quad \stackrel{\circ}{\Gamma}_{\mu v}^{\rho} \equiv \frac{1}{2} g^{\rho \sigma}\left(\partial_{\mu} g_{v \sigma}+\partial_{v} g_{\sigma \mu}-\partial_{\sigma} g_{\mu v}\right)
$$

whose transformation law is given by

$$
\tilde{\Gamma}_{\mu \nu}^{\rho}=\frac{\bar{\phi}}{\phi} \bar{\Gamma}_{\lambda \varepsilon}^{\sigma} \frac{\partial x^{\rho}}{\partial \bar{x}^{\sigma}} \frac{\partial \bar{x}^{\lambda}}{\partial x^{\mu}} \frac{\partial \bar{x}^{\varepsilon}}{\partial x^{\nu}}+\frac{1}{\phi} \frac{\partial x^{\rho}}{\partial \bar{x}^{\sigma}} \frac{\partial^{2} \bar{x}^{\sigma}}{\partial x^{\mu} \partial x^{\nu}}+\frac{1}{\phi} \delta_{v}^{\rho} \frac{\partial}{\partial x^{\mu}} \ln \left(\frac{\bar{\phi}}{\phi}\right)
$$

One can define the covariant derivative for a vector field as

$$
\nabla_{\mu} A^{v} \equiv \frac{1}{\phi} \partial_{\mu} A^{v}+\tilde{\Gamma}_{\mu \alpha}^{v} A^{\alpha}, \quad \nabla_{\mu} A_{v} \equiv \frac{1}{\phi} \partial_{\mu} A_{v}-\tilde{\Gamma}_{\mu \nu}^{\alpha} A_{\alpha} .
$$

The richness of the Lyra's geometry is demonstrated by the curvature [3]

$$
\tilde{R}_{\beta \alpha \sigma}^{\rho} \equiv \frac{1}{\phi^{2}}\left(\frac{\partial\left(\phi \tilde{\Gamma}_{\alpha \sigma}^{\rho}\right)}{\partial x^{\beta}}-\frac{\partial\left(\phi \tilde{\Gamma}_{\beta \sigma}^{\rho}\right)}{\partial x^{\alpha}}+\phi \tilde{\Gamma}_{\beta \lambda}^{\rho} \phi \tilde{\Gamma}_{\alpha \sigma}^{\lambda}-\phi \tilde{\Gamma}_{\alpha \lambda}^{\rho} \phi \tilde{\Gamma}_{\beta \sigma}^{\lambda}\right)
$$

and the torsion [15]

$$
\tilde{\tau}_{\mu \nu}{ }^{\rho} \equiv \tilde{\Gamma}_{\mu \nu}^{\rho}-\tilde{\Gamma}_{\nu \mu}^{\rho}-\frac{1}{\phi}\left(\delta_{\mu}^{\rho} \partial_{v}-\delta_{v}^{\rho} \partial_{\mu}\right) \ln \phi
$$

where the second term is the anholonomic contribution, thus, we get

$$
\tilde{\tau}_{\mu v}^{\rho}=-\frac{1}{\phi}\left(\delta_{\mu}^{\rho} \partial_{v}-\delta_{v}^{\rho} \partial_{\mu}\right) \ln \bar{\phi}, \quad \tilde{\tau}_{\mu} \equiv \tilde{\tau}_{\mu \rho}^{\rho}=\frac{3}{\phi} \partial_{\mu} \ln \bar{\phi} .
$$




\section{The massive DKP field in Lyra manifold}

In Minkowski space-time the massive DKP theory is given by the following Lagrangian density

$$
\mathcal{L}=\frac{i}{2} \bar{\psi} \beta^{a} \partial_{a} \psi-\frac{i}{2} \partial_{a} \bar{\psi} \beta^{a} \psi-m \bar{\psi} \psi
$$

where $\bar{\psi}=\psi^{\dagger} \eta^{0}, \eta^{0}=2\left(\beta^{0}\right)^{2}-1$, and the $\beta^{a}$ are matrices satisfying the massless DKP algebra ${ }^{1}$

$$
\beta^{a} \beta^{b} \beta^{c}+\beta^{c} \beta^{b} \beta^{a}=\beta^{a} \eta^{b c}+\beta^{c} \eta^{b a}
$$

The resulting equation of motion for the DKP field $\psi$ is

$$
i \beta^{a} \partial_{a} \psi-m \psi=0
$$

The above equations can be generalized to Lyra space-time [2] $\mathbb{L}$ through the formalism of tetrads (or vierbeins) together the minimal coupling procedure [16, 17]. Here we shall simply quote the main results we need. For details, in Riemann and Riemann-Cartan manifolds, we refer respectively to [7, 8] and [9, 10, 12] and references therein.

We consider a Lyra space-time $\mathbb{L}$ with metric $g_{\mu v}$, whose point coordinates are labelled $x^{\mu}$. To each point in $\mathbb{L}$ we attach a Minkowski space-time $\mathbb{M}$ with metric $\eta_{a b}$, whose point coordinates are labelled $x^{a}$. The DKP fields $\psi$ are Lorentz group representations in Minkowski space-time. The projections into $\mathbb{L}$ of all tensor quantities defined on $\mathbb{M}$ are done via the tetrad fields $e_{a}^{\mu}(x)$ :

$$
g_{\mu v}(x)=\eta_{a b} e_{\mu}^{a}(x) e_{v}{ }^{b}(x), \quad e_{v}{ }^{a} e^{v}{ }_{b}=\delta_{b}^{a}, \quad e=\operatorname{det}\left(e_{\mu}{ }^{a}\right)=\sqrt{-g},
$$

where $g=\operatorname{det}\left(g_{\mu v}\right)$.

The resulting action for massive DKP fields minimally coupled to Lyra's manifold is

$$
S_{D K P}=\int d^{4} x \phi^{4} e\left(\frac{i}{2} \bar{\psi} \beta^{a} e^{\mu}{ }_{a} \nabla_{\mu} \psi-\frac{i}{2} \nabla_{\mu} \bar{\psi} e^{\mu}{ }_{a} \beta^{a} \psi-m \bar{\psi} \psi\right)
$$

where $\nabla_{\mu}$ is the Lyra covariant derivative associated to the affine connection $\tilde{\Gamma}_{\alpha \mu}^{v}$.

The covariant derivatives of DKP fields are

$$
\nabla_{\mu} \psi=\frac{1}{\phi} \partial_{\mu} \psi+\frac{1}{2} \omega_{\mu a b} S^{a b} \psi \quad, \quad \nabla_{\mu} \bar{\psi}=\frac{1}{\phi} \partial_{\mu} \bar{\psi}-\frac{1}{2} \omega_{\mu a b} \bar{\psi} S^{a b}
$$

where $S^{a b}=\left[\beta^{a}, \beta^{b}\right]$ and $\omega_{\mu a b}$ is the spin connection.

The Euler-Lagrange equation for the $\psi$ field is

$$
i \beta^{\mu}\left(\nabla_{\mu}+\frac{1}{2} \tilde{\tau}_{\mu}\right) \psi-m \psi=0
$$

where we have used the metricity condition, $\nabla_{\alpha} e_{\mu}{ }^{a}=\frac{1}{\phi} \partial_{\alpha} e_{\mu}{ }^{a}-\tilde{\Gamma}^{\rho}{ }_{\alpha \mu} e_{\rho}{ }^{a}+\omega_{\alpha b}{ }^{a} e_{\mu}{ }^{b} \equiv 0$.

\footnotetext{
${ }^{1}$ We choose a representation in which $\beta^{0^{\dagger}}=\beta^{0}, \beta^{i \dagger}=-\beta^{i}$.
} 


\subsection{The scalar sector}

In Minkowski space-time, the "projectors" $P$ and $P^{a}$ select the spin 0 sector of the theory (see [13, 7]) such that $P \psi$ is a scalar and $P^{a} \psi$ is a vector field. Thus, from these projectors defined in $\mathbb{M}$ we can construct the projectors in Lyra manifold as $P^{\mu}=e^{\mu}{ }_{a} P^{a}=e^{\mu}{ }_{a} P \beta^{a}=P \beta^{\mu}$.

From the definitions above and the properties of $P$ and $P^{a}$ it is easy to verify that $P^{\mu} \beta^{\nu}=$ $P g^{\mu \nu}, P S^{\mu v}=0$, and it can also be seen that $P \nabla_{\mu} \psi=\nabla_{\mu}(P \psi)$ and $P^{v} \nabla_{\mu} \psi=\nabla_{\mu}\left(P^{v} \psi\right)$ due to the metricity condition. Therefore, under general coordinate transformations, $P \psi$ is a scalar and $P^{v} \psi$ is a vector.

By applying the projectors $P$ and $P^{\mu}$ to the equation (3.6), we get respectively,

$$
m P \psi=i\left(\nabla_{\mu}+\frac{1}{2} \tilde{\tau}_{\mu}\right) P^{\mu} \psi, \quad m P^{\mu} \psi=i\left(\nabla^{\mu}+\frac{1}{2} \tilde{\tau}^{\mu}\right) P \psi
$$

by mixing both equation, we obtain the equation of motion for the scalar $P \psi$. We choose a representation where DKP field is a 5-vector column such as $\psi=\left(\varphi, \psi^{0}, \psi^{1}, \psi^{2}, \psi^{3}\right)^{T}, P \psi=(\varphi, 0,0,0,0)^{T}$ and $P^{a} \psi=\left(\psi^{a}, 0,0,0,0\right)^{T}$. Thus, we have

$$
\left(\nabla_{\mu}+\frac{1}{2} \tilde{\tau}_{\mu}\right)\left(\nabla^{\mu}+\frac{1}{2} \tilde{\tau}^{\mu}\right) \varphi+m^{2} \varphi=0
$$

As we can see above, the interaction with torsion does not disappear, even after we selected the spin 0 sector of the DKP field. This interaction is present both in the connection $\tilde{\Gamma}_{\alpha \mu}^{v}$ used in the calculation of the covariant derivative $\nabla_{\mu}$ and in the explicit presence of terms containing the trace torsion $\tilde{\tau}_{\mu}$ in the equation above.

On the other hand, when the Lyra geometry is minimally coupled to the massive KleinGordon-Fock field, we get

$$
S_{K G}^{\mathbb{L}}=\int d^{4} x \phi^{4} \sqrt{-g}\left(\nabla_{\mu} \varphi^{*} \nabla^{\mu} \varphi-m^{2} \varphi^{*} \varphi\right),
$$

where the covariant derivative of the KGF scalar reads $\nabla_{\mu} \varphi=\frac{1}{\phi} \partial_{\mu} \varphi$.

The KGF action (3.9) results in the following equation of motion

$$
\left(\nabla_{\mu}+\tilde{\tau}_{\mu}\right) \nabla^{\mu} \varphi+m^{2} \varphi=0
$$

We can see that there exist interaction with the trace torsion. It is a different situation to what happened in Riemann-Cartan spacetime where the scalar field does not couple with torsion [9]. However, the spin 0 DKP equation (3.8) is different of KGF equation (3.10).

The difference will be better understood if we project the DKP action (3.5) to its spin 0 sector. Thus, by using the equation (3.7) to relate the vector $\psi^{\mu}$ to the scalar $\varphi$, and after some integration by parts and a rescaling $\varphi \rightarrow \sqrt{m} \varphi$, the DKP action (3.5) reads as

$$
S_{D K P 0}^{\mathbb{L}}=\int d^{4} x \phi^{4} \sqrt{-g}\left(\nabla_{\mu} \varphi^{*} \nabla^{\mu} \varphi-m^{2} \varphi^{*} \varphi-\frac{1}{2} \nabla_{\mu} \tilde{\tau}^{\mu} \varphi \varphi^{*}-\frac{1}{4} \tilde{\tau}^{\mu} \tilde{\tau}_{\mu} \varphi^{*} \varphi\right)
$$

from this action we can obtain the spin 0 DKP equation given in (3.8). And it has two non minimal coupling which do not appear in the KGF action (3.9). 


\subsection{The vectorial sector}

Now we use the Umezawa's "projectors" $R^{\mu}$ and $R^{\mu v}$ in order to analyze the spin 1 sector of the theory. We remember that $R^{\mu} \psi \equiv \psi^{\mu}$ is a vector and $R^{\mu v} \psi \equiv \psi^{\mu v}$ is a second rank antisymmetric tensor in a Lyra sense. Applying these operators on the equation of motion (3.3) we get, respectively,

$$
m \psi^{\mu}=i\left(\nabla_{\beta}+\frac{1}{2} \tilde{\tau}_{\beta}\right) \psi^{\mu \beta}, \quad m \psi^{\mu \beta}=i\left(\nabla_{\alpha}+\frac{1}{2} \tilde{\tau}_{\alpha}\right)\left(g^{\alpha \beta} \psi^{\mu}-g^{\alpha \mu} \psi^{\beta}\right)
$$

by mixing both equations, we found the equation of motion for the vector field $\psi^{\mu}$

$$
\left(\nabla_{\beta}+\frac{1}{2} \tilde{\tau}_{\beta}\right)\left(\nabla_{\alpha}+\frac{1}{2} \tilde{\tau}_{\alpha}\right)\left(g^{\alpha \beta} \psi^{\mu}-g^{\alpha \mu} \psi^{\beta}\right)+m^{2} \psi^{\mu}=0
$$

We project the massive DKP action (3.5) to its spin 1 sector. Then, by using the equation (3.12) which relates the tensor field $\psi^{\mu v}$ to the vector $\psi^{\mu}$ and, after some integration by parts and a rescaling $\psi^{\mu} \rightarrow \sqrt{m} \psi^{\mu}$ the DKP action (3.5) becomes

$$
\begin{aligned}
S_{D K P 1}^{\mathbb{L}}=\int d^{4} x \phi^{4} e( & -\frac{1}{2 \phi^{2}} f^{\mu v} f_{\mu v}^{*}+m^{2} \psi_{\mu}^{*} \psi^{\mu}-\frac{1}{6 \phi}\left(f^{\mu v} \Sigma_{\mu v}^{*}+f_{\mu v}^{*} \Sigma^{\mu v}\right)-\frac{1}{18} \Sigma^{\mu v} \Sigma_{\mu v}^{*}+ \\
& \left.-\frac{1}{3 \phi}\left(f^{* \mu v} S_{\mu v}+S^{* \mu v} f_{\mu v}\right)-\frac{1}{9}\left(\Sigma^{* \mu v} S_{\mu v}+S^{* \mu v} \Sigma_{\mu v}\right)-\frac{2}{9} S^{* \mu v} S_{\mu v}\right)
\end{aligned}
$$

where

$$
f_{\mu v} \equiv \partial_{\mu} \psi_{v}-\partial_{v} \psi_{\mu}, \quad \Sigma_{\mu v} \equiv \frac{1}{2}\left(\tilde{\tau}_{\mu} \psi_{v}-\tilde{\tau}_{v} \psi_{\mu}\right), \quad S_{\mu v} \equiv \frac{3}{2 \phi}\left(\psi_{v} \partial_{\mu}-\psi_{\mu} \partial_{v}\right) \ln (\phi)
$$

Otherwise, the Proca's lagrangian in Minkowski space-time is given by

$$
\mathcal{L}_{P R}=-\frac{1}{2}\left(\partial_{a} A_{b}^{*}-\partial_{b} A_{a}^{*}\right)\left(\partial^{a} A^{b}-\partial^{b} A^{a}\right)+m^{2} A_{a}^{*} A^{a}
$$

By making the minimal coupling procedure to the Lyra spacetime, we get

$$
\begin{aligned}
S_{P R}^{\complement}=\int d^{4} x \phi^{4} e(- & \frac{1}{2 \phi^{2}} F^{\mu v} F_{\mu v}^{*}+m^{2} A_{\mu}^{*} A^{\mu}+\frac{1}{3 \phi}\left(F^{\mu v} \Sigma_{\mu v}^{*}+F_{\mu v}^{*} \Sigma^{\mu v}\right)-\frac{2}{9} \Sigma^{\mu v} \Sigma_{\mu v}^{*}+ \\
& \left.-\frac{1}{3 \phi}\left(F^{* \mu v} S_{\mu v}+S^{* \mu v} F_{\mu v}\right)+\frac{2}{9}\left(\Sigma^{* \mu v} S_{\mu v}+S^{* \mu v} \Sigma_{\mu v}\right)-\frac{2}{9} S^{* \mu v} S_{\mu v}\right)
\end{aligned}
$$

\section{Comments}

A simple comparison between the Lagrangians and equations of motion shows us the unequivalence of DKP theory with KGF and Proca descriptions of scalar and vector massive particles. However, a more accurated inspection reveals the spin 0 case as a problem of nonminimal coupling. In the spin 1 case the situation is more complicated, because all terms in DKP Lagrangian are also present in the Proca, but with modified coupling constants.

Now, from (3.14) we can see that

$$
S_{D K P 1}^{M_{4}}=\int d^{4} x\left[-\frac{1}{2}\left(\partial_{a} \psi_{b}^{*}-\partial_{b} \psi_{a}^{*}\right)\left(\partial^{a} \psi^{b}-\partial^{b} \psi^{a}\right)+m^{2} \psi_{a}^{*} \psi^{a}\right] \quad \longrightarrow \quad S_{D K P 1}^{\mathbb{L}}
$$


by the prescription

$$
\partial_{a} \rightarrow D_{\mu} \equiv \nabla_{\mu}+\frac{1}{2} \tau_{\mu}
$$

One can see that the prescription (4.2) only changes the strength of the coupling with respect the usual minimal coupling procedure. It can be explicitly observed by analysing the proportion between the coefficients of the interactions in the actions $S_{D K P 1}^{\mathbb{L}}(3.14)$ and $S_{P R}^{\mathbb{L}}(3.17)$.

In our future perspectives we will do a study of the relationship between Lyra geometry and gauge theories, which is now in course using the Utiyama general theory. At same time, the coupling of Dirac field with this manifold is in preparation. We hope that these studies can clarify if the nonequivalence is restricted to manifolds with torsion and curvature, or if it is related to the structure of the field theory used to describe the particles.

\section{Acknowledgements}

R. C. and C. A. M. M. thank FAPESP (grants 01/12611-7 and 01/12584-0 respectively) for support. B. M. P. thanks CNPq and FAPESP (grant 02/00222-9) for partial support.

\section{References}

[1] H. Weyl - Gravitation und Elektrizität, Sitzungsber. Preuss. Akad. Wissensch. (1918) 465 ; Reprinted (english version) in: L. O’Raifeartaigh, The Dawning of Gauge Theory, Princeton Series in Physics, Princenton 1997.

[2] G. Lyra, Über eine Modifikation der Riemannschen Geometrie, Math. Z. 54 (1951) 52; E. Scheibe, Über einen verallgemeinerten affinen Zusammenhang, Math. Z. 57 (1952) 65.

[3] D. K. Sen and J. R. Vanstone, On Weyl and Lyra Manifolds, J. Math. Phys. 13 (1972) 990.

[4] A. Pradhan and H. R. Pandey, Bulk Viscous Cosmological Models in Lyra Geometry gr-qc/0307038.

[5] G. S. Khadekar and A. R. Nagpure, Higher Dimensional Static Cosmological Model in Lyra Manifold gr-qc/0111096.

[6] A. Pradhan, I. Iotemshi and G.P. Singh, Plane Symmetric Doamin Wall in Lyra Geometry, Astrophys. Space Sci. 288 (2003) 315.

[7] J. T. Lunardi, B. M. Pimentel and R. G. Teixeira, Duffin-Kemmer-Petiau equation in Riemannian space-times, in Geometrical Aspects of Quantum Fields, Proceedings of the 2000 Londrina Workshop, Londrina, Brazil, ed. A. A. Bytsenko, A. E. Gonçalves and B. M. Pimentel, World Scientific, Singapore 2001 [gr-qc/9909033].

[8] R. Casana, J. T. Lunardi, B. M. Pimentel and R. G. Teixeira, Int. J. Mod. Phys. A 17 (2002) 4197.

[9] J. T. Lunardi, B. M. Pimentel and R. G. Teixeira, Gen. Rel. Grav. 34 (2002) 491.

[10] R. Casana, J. T. Lunardi, B. M. Pimentel and R. G. Teixeira, Gen. Rel. Grav. 34 (2002) 1941.

[11] Harish-Chandra, Proc. Roy. Soc. Lond. A 186 (1946) 502.

[12] R. Casana, V. Ya. Fainberg, J. T. Lunardi, B. M. Pimentel and R. G. Teixeira, Class. Quantum Grav. 20 (2003) 2457.

[13] H. Umezawa, Quantum Field Theory, North-Holland, Amsterdam 1956.

[14] R. A. Krajcik and M. M. Nieto, Am. J. Phys. 45 (1977) 818.

[15] F. De Felice and C. J. S. Clarke, Relativity on curved Manifolds, Cambridge Monographs on Mathematical Physics, Cambridge University Press, Cambridge 1990.

[16] F. W. Hehl, P. von der Heyde and G. D. Kerlick, Rev. Mod. Phys. 48 (1976) 393.

[17] V. De Sabbata and M. Gasperini, Introduction to Gravitation, World Scientific, Singapore 1985. 